\title{
Pharyngitis, Laryngitis, Tracheitis
}

\author{
Eine akute Pharyngitis ebenso wie eine Laryngitis, die bei viralen \\ oder bakteriellen Infektionen der Atemwege oder infolge von Rauchen \\ bzw. dem Aufenthalt in trockener Luft auftreten, können gut mit \\ Phytotherapeutika behandelt werden. Auch bei einer Tracheitis infolge von \\ viralen oder bakteriellen Infektionen, Rauchen oder nach Tracheotomie \\ kann Phytotherapie mit Erfolg eingesetzt werden.
}

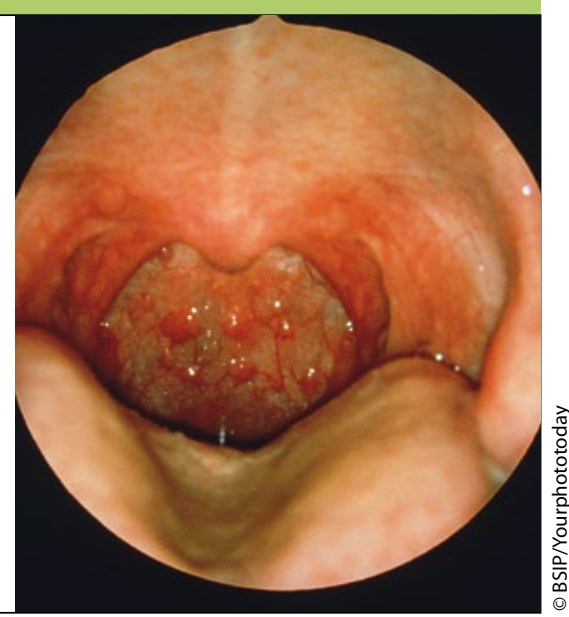

— Die Wirksamkeit von Phytotherapeutika bei Pharyngitis, Laryngitis und Tracheitis beruht auf antiphlogistischen, antibakteriellen, antiviralen und lokal anästhesierenden Wirkungen.

- Pharyngitis: Empfohlen werden Spülund Gurgellösungen mit Auszügen aus Kamillenblüten oder Salbeiblättern, zudem Kombinationspräparate, die weitere ätherische Öle z. B. aus Thymiankraut, Gewürznelke, Anisfrüchten enthalten können. Außerdem können Zubereitungen aus Spitzwegerichkraut schluckweise getrunken werden. Wichtig ist die mehrmals tägliche Anwendung dieser Maßnahmen.

Zum Feuchthalten der gereizten Schleimhäute können Salbeibonbons oder Pastillen mit Isländischem Moos

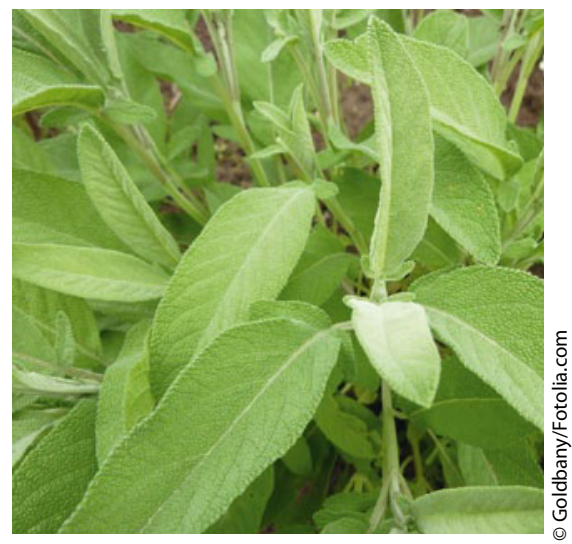

Salbei enthält eine Vielzahl ätherischer Öle, die u. a. antibakteriell und antiviral wirken. mehrmals täglich je nach Bedarf langsam gelutscht werden. Eine Kombination mit anderen Schleimstoffdrogen wie Malvenblüten, Malvenblättern oder Eibischwurzel ist sinnvoll. Zubereitungen aus der Wurzel von Pelargonium sidoides zur oralen Anwendung sind ebenfalls für die Behandlung von akuter Pharyngitis, insbesondere einer Tonsillopharyngitis geeignet.

- Laryngitis: Zur Behandlung können Zubereitungen mit Isländischem Moos, Kamillenblüten und Salbeiblättern eingesetzt werden, Anwendung wie oben beschrieben. Zur Prävention einer Laryngitis, z. B. bei Rednern oder Sängern, eignen sich Zubereitungen aus Isländischem Moos oder Salbeiblättern.

- Tracheitis: Sie kann mit Zubereitungen aus Kamillenblüten oder Salbeiblättern behandelt werden.

Alle phytotherapeutischen Maßnahmen sind sehr gut verträglich und können allein oder adjuvant zu chemischsynthetischen Arzneimitteln verwendet werden. Alkoholische Salbeiextrakte und reines Salbeiöl sollten jedoch wegen ihres Thujongehaltes während Schwangerschaft und Stillzeit nicht eingenommen werden.

\section{Mindestens zwei Liter pro Tag trinken}

Als weitere Maßnahmen, die mit der genannten Phytotherapie kombiniert werden können, ist bei akuter Pharyngitis und Laryngitis auch Hydrotherapie indiziert wie ansteigende Arm- und Fußbäder, aber auch Inhalationen mit 1- bis 2\%iger Sole oder Emser Salz. Eine wichtige Begleitmaßnahme ist reichliches Trinken (mindestens zwei Liter pro Tag), wobei besonders Tee aus Thymiankraut, Salbeiblättern und Kamillenblüten empfohlen werden kann. Auf scharf gewürzte Speisen und unverdünnte Obstsäfte sollte verzichtet werden, da sie die Schleimhaut zusätzlich reizen.

\section{Abhärtungsmaßnahmen gegen chronische Pharyngitis}

Bei chronischer Laryngitis können, nachdem eine sorgfältige Diagnostik zum Ausschluss spezifisch therapierbarer Ursachen erfolgt ist, ebenfalls die bereits ausgeführten phytotherapeutischen Maßnahmen empfohlen werden. Zusätzlich sollten Abhärtungsmaßnahmen durchgeführt werden. Aus der Hydrotherapie eignen sich dafür Körperbürstungen, wechselwarme Waschungen, wechselwarme Duschen, ansteigende Armbäder, Brustwickel, ansteigende und wechselwarme Fußbäder, Arm- und Kniegüsse, Wassertreten, Tautreten und Sauna. Ebenfalls zu empfehlen sind Sport an frischer Luft sowie im Rahmen der Klimatherapie jährliche Aufenthalte an Nord- oder Ostsee.

- Prof. Dr. med. Karin Kraft, Lehrstuhl Naturheilkunde, Universität Rostock 